\title{
Benign Metastasizing Leiomyoma of the Lung: Lesion Regression after Surgical Ovariectomy
}

\author{
Rocco Delfanti*1, Luigi Conti ${ }^{1}$, Claudia Biasini ${ }^{2}$ and Patrizio Capelli ${ }^{1}$ \\ ${ }^{1}$ Department of Surgery, G Da Saliceto Hospital, Piacenza, Italy \\ ${ }^{2}$ Department of Oncology, G. Da Saliceto Hospital, Piacenza, Italy
}

Received: February 19, 2018; Published: February 26, 2018

*Corresponding author: Luigi Conti, Department of General, Vascular and Breast Surgery, Piacenza, Italy. Tel: +39 3334043148; Fax +39 0523303173 , Email: dr.luigiconti@gmail.com

\begin{abstract}
Benign metastasizing leiomyomas are a rare disease occurring in the lung of pre-menopausal women with a history of benign leiomyoma of the uterus; they are discovered incidentally because often asymptomatic and should be differentiated from low grade uterus sarcoma. Tumor cells are positive for estrogen and progesterone receptors so therapy is based on ovarian hormonal suppression, performed chemically or by surgical ovariectomy. We present the case of a 40-year old woman with a history of benign uterine leiomyoma who was admitted to the Oncological Department to investigate the presence of solid nodules in lungs, found accidentally on chest radiogram. She underwent to hysterectomy and bilateral ovariectomy; specimen confirmed only the persistence of benign uterine leiomyoma.
\end{abstract}

No further therapy was proposed and the follow up was managed with clinical visit and thoracic CT scan every six months for the first year, twelve monthly then. BML is a rare disease, reported as single cases occasionally in the literature. Lungs are the organs most often involved and radiologically the lesions tend to present as multiple, bilateral, well circumscribed solid nodules of various dimensions. Interesting is the significant response in the regression of dimension and number of lung nodules, due only to hormonal suppression and the persistence of the response along a period of four years, this confirming both his benign nature and the influence of ovarian hormonal activity on BML cells.

Keywords: Benign Metastasizing Leiomyoma-lung-uterine leiomyoma

Abbreviations: BML: Benign Metastasizing Leiomyoma; CT: Computed Tomography; LGUS: Low Grade Uterus Sarcoma ;VATS: Video-Assisted Thoracic Surgery

\section{Introduction}

BML is a rare disease occurring in the lungs of pre-menopausal women with a history of benign leiomyoma of the uterus [1]. The tumors are usually discovered incidentally, rarely associated with clinical symptoms. Low grade uterus sarcoma (LGUS) are more often suspected and diagnosis between BML and LGUS is made only on surgical specimens. Tumor cells are positive for estrogen and progesterone receptors so therapy is based on ovarian hormonal suppression, performed chemically or by surgical ovariectomy.

\section{Case Presentation}

A 40-year old woman, with a non-smoking habit, was admitted to the Ontological Department for further examinations on solid nodules in both lungs, found accidentally on chest radiogram. The patient was nulliparous, but she underwent to surgical myomectomy for uterine leiomyomas four and six years before. Blood tests were normal and tumor markers were negative. A CT scan of the chest showed a total of about 30 multiple solid nodules in both lungs, ranging from size of few millimeters to a maximum of $8 \mathrm{~cm}$ in diameter. LGUS was suspected and VATS biopsy was performed to confirm the diagnosis; three nodules of about $1 \mathrm{~cm}$ in diameter were resected from the right lung. The patient was discharged uneventfully on fourth post-operative day. Pathological examination on the specimens revealed a proliferation of spindle shaped smooth muscle cells, in addition nodules containing epithelial-line clefts representing entrapped respiratory epithelium.

Tumor cells were histochemically positive for muscle Actin, desmin, caldesmin, CD 10, estrogen and progesterone receptors; the Ki-67 activity rate was low, about $3 \%$. The lack of necrosis, low mitotic activity, well differenced appearance on cytology and low proliferation activity oriented the diagnosis to BML instead of LGUS. To confirm the diagnosis of BML the histopathology on previous uterus specimens was reviewed, confirming the diagnosis of benign uterus leiomyoma. Both pharmacological and surgical castration was proposed to the patient. She decided for hysterectomy and 
bilateral ovariectomy in other institution; specimen confirmed only the persistence of benign uterine leiomyoma. No further therapy was proposed and the follow up was managed with clinical visit and thoracic CT scan every six months for the first year, twelve monthly then. During the first year the number and dimension of the lesions decreased of more than $50 \%$, so the most part of small lesions disappeared on CT scan. This radiological regression keep stable in the following three years and the patient laid asymptomatic without any further therapy (Figures $1 \& 2$ ).

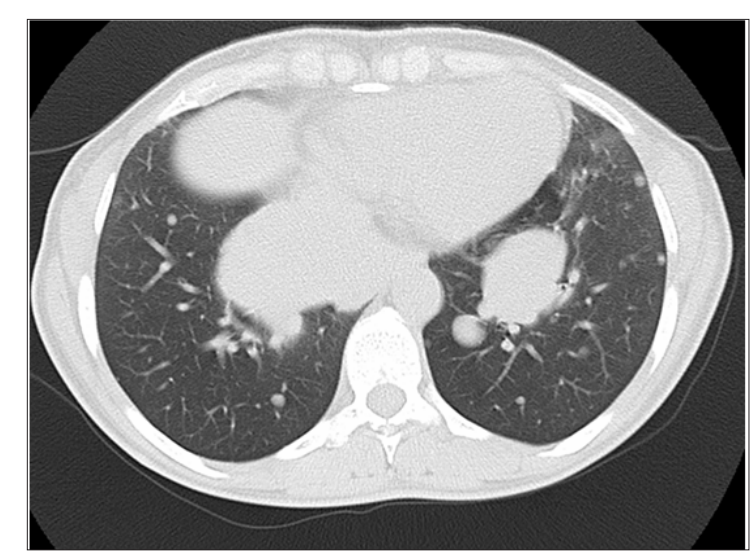

Figure 1: Pre-surgerythoracic CT-scan.

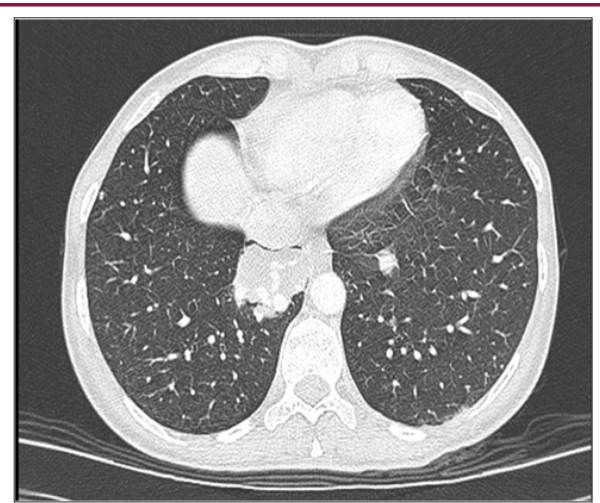

Figure 2: After 4 year a control CT scan of the thorax revealed that the nodules of the inferior lobes were both reduced in size.

\section{Discussion}

BML is a rare disease, reported as single cases occasionally in the literature. Lungs are the organ most often involved and radio logically the lesions tend to present as multiple, bilateral, well circumscribed solid nodules of various dimensions [2]. Previous or present history of uterus leiomyoma is needed for diagnosis [3]. But the most frequent LGUS must be suspected; so definitive diagnosis can be achieved only on surgical specimens. Lack of necrosis, well differenced appearance on cytology, immune staining, positive ovarian hormonal receptors and low Ki-67 led to the diagnosis. As resection of all pulmonary nodules is not feasible in the most part of the cases in literature, ovarian hormonal suppression is usually performed after diagnosis, with stabilization of the disease in literature reported cases. Interesting in our report is the significant response in the regression of dimension and numbers of lung nodules, due only to hormonal suppression and the persistence of the response along a period of four years [4]. This confirming both his benign nature and the influence of ovarian hormonal activity on LBM cells [5]. However, the rarity of LBM allows only case reports, getting more difficult the confirmation of these arguments on pathogenesis and treatment of LBM.

\section{References}

1. Kayser K, Zink S, Schneider T, Dienemann H, André S, et al. (2000) Benign metastasizing leiomyoma of the uterus: documentation of clinical, immunohistochemical and lectin-histochemical data of ten cases. Virchows Arch 437(3): 284-292.

2. Abramson S, Gilkeson RC, Goldstein JD, Woodard PK, Eisenberg R, et al. (2001) Benign metastasizing leiomyoma: clinical, imaging, and pathologic correlation. Am J Roentgenol 176(6): 1409-1413.

3. Miller J, Shoni M, Siegert C, Lebenthal A, Godleski J, et al. (2016) Benign metastasizing leiomyomas to the lungs: an institutional case series and a review of the recent literature. Ann Thorac Surg 101(1): 253-258.

4. Steiner PE (1939) Metastasizing fibroleiomyoma of the uterus: report of a case and review of the literature. Am J Pathol 15(1): 89-107.

5. Rao AV, Wilson J, Sylvester K (2008) Pulmonary benign metastasizingleiomyoma following hysterectomy: a clinicopathologiccorrelation. J Thorac0ncol 3(6): 674-676.

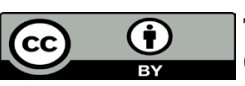

This work is licensed under Creative Commons Attribution 4.0 License

Submission Link: http://biomedres.us/submit-manuscript.php

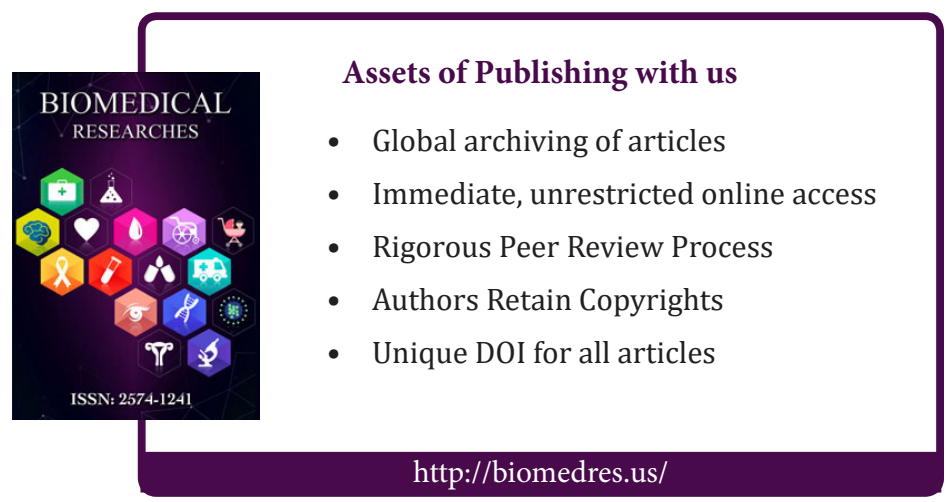

